\title{
A HYBRID COMPRESSION MODEL FOR CLUSTERS OF SIMILAR MEDICAL IMAGES
}

\author{
JIANN-DER LEE ${ }^{1}$, SHU-YEN WAN ${ }^{2}$ AND RUI-FENG WU ${ }^{1}$ \\ ${ }^{1}$ Department of Electrical Engineering, \\ ${ }^{2}$ Graduate Institute of Information Engineering, \\ Chang Gung University, Tao-Yuan, Taiwan
}

\begin{abstract}
In this paper, a new compression scheme called hybrid compression model (HCM) is proposed for compressing clusters of similar images. The HCM exploits region growing to segment the median image that created by a cluster of similar images; and further, it uses centroid method to predict the values of original images data. The difference between the predict values and original data is stored for later use of progressive transmission. The experimental results obtained on various images show that our method provides significant improvement in compression efficiency while compared to the traditional centroid method.
\end{abstract}

Biomed Eng Appl Basis Comm, 2003 (February); 15: 38-45.

\section{INTRODUCTION}

Compressing an image cluster with multiple slices is very important in a telemedicine system, especially radiology, because the most commonly used digital modalities, including magnetic resonance (MR), computed tomography (CT), positron emission tomography (PET), generate multiple slices in a single examination. Multiple slices generated this way are normally anatomically or physiologically correlated to each other. In other words, there are some image structure similarities between adjacent slices. Although it is possible to compress an image cluster slice by slice, more efficient compression can be achieved by exploring the correlation between slices. Methods to remove slice correlation for clusters of similar images include threedimension (3-D) transforms and prediction methods. In this paper, we will focus on the latter approach, i.e., prediction methods.

In general, there are two categories for image compression, i.e., lossless and lossy [1]. Lossless com-

Received: Aug. 15, 2002; Accepted: Feb. 8, 2003

Correspondance: Jiann-Der Lee, Professor

Department of Electrical Engineering, Chang Gung University, Tao-Yan, Taiwan. pression is an error free compression. The decompressed image is all the same as the original image, on a pixel-by-pixel basis. In spite of this benefit, only a small amount of compression is possible and hence, the total compression ratio is low as expected. In general, for some applications, the lossless compression is the only acceptable method of image compression. These kinds of applications tolerate no loss in information. The typical examples consist of 1) the archival of medical images where the loss of any information is not tolerated because it may affect the accuracy of the diagnosis, 2) the archival of business document where losing information is illegal. It is known that lossless image compression can provide a compression ratio ranging between 2 and 10 .

On the other hand, lossy compression method is a kind of irreversible compression. It is based on compressing the accuracy of the recovered image in exchange for more compression, and usually the reconstructed image contains the distortion, which may or may not be visually apparent. If this resulting degradation can be tolerated for some specific applications, the compression ratio can be significant improved with easy. To our knowledge, a lot of lossy compression approaches can often produce good recognizable images with compression ratios of 30 or higher.

In the past, several research efforts applied the prediction method to image compression [1-3]. 
Karadimitriou [4] proposes a similar images compression model called Enhanced Compression Model, which exploits centroid method to reduce cluster redundancy and improves performance even more. Moreover, progressive transmission [5,6] plays an important role in this field for its compression efficiency, speed and bit rate less than 1 bit per pixel (bpp). The nature of the pyramid structure is ideal for progressive transmission. The pyramid decomposition of an image consists of an original image and successively lower resolution images. Roos et al. [7] proposed a prediction/residual approach to form the variable resolution pyramid by subsampling. This kind of approach has been termed hierarchical interpolation (HINT).

In this paper, a new hybrid compression model (HCM) is proposed for compressing clusters of similar images such as medical images. HCM is a segmentation based prediction method. It combines region growing and centroid method to predict the values of original images data, and the difference between the predict values and original data is stored for later use in the stage of progressive transmission. In additions, HINT is also used in progressive transmission procedure due to its excellent performance in hierarchical decomposition.

This paper is organized as follows. Section II gives a brief introduction on the similar images compression that includes cluster redundancy and centroid method. The proposed compression model is illustrated in Section III. Section IV presents experimental results and the conclusion is summarized in Section V.

\section{THE PROPOSED HYBRID COM- PRESSION MODEL}

\subsection{Centroid Method}

Predictive decorrelation is an important technique for medical image compression. In predictive decorrelation, an estimate of the image based on previously encoded pixels or on a statistical model of the image is subtracted from the original image. The recorded differences will be small when the estimate agrees well with the original image. A differential image containing mostly small values can be efficiently coded by a variable-length code. Several methods such as DPCM [7] and adaptive DPCM are often used.

For a cluster of $K$ images with $N$ pixels per image, the formula for predicting the value of a pixel using the average value of the same position across the cluster of similar image and a correlation term can be expressed as below, i.e.,

$$
\begin{gathered}
C_{i+1, j}=m_{i+1}+e_{i, j} \\
e_{i, j}=x_{i, j}-m_{i}
\end{gathered}
$$

where $C_{i+1, j}$ is an estimate at position $i+1$ in image $j$, $x_{i, j}$ is the pixel at position $i$ in image $j$, and $m_{i}$ is the average value form position $i$ across all images. Eq. (1) is so called centroid method [4], and it can help to reduce inter-image redundancy and then improve compression efficiency. In this paper, the proposed scheme is based on similar concept of the centroid model, but takes the advantage of median model and image segmentation techniques, to reduce the cluster redundancy more efficiently. Using median value but average value can avoid the influence of the extreme pixel value due to the noise and reduce the diversion of a pixel cluster.

\subsection{Region Growing Algorithm}

In general, region growing techniques [8] are often used for object identification or features analysis. However, the main purpose of region growing in this approach is to group spatially connected pixels whose gray level are within a small dynamic range.

There are two criteria required for consideration while applying region growing procedure. The first one is that the neighbor pixel is not a member of any regions already grown, and the other one is the absolute difference between the neighbor pixel and the corresponding center pixel is less than error level:

$$
\text { error level }=2^{\text {error bits- } 1}
$$

where error bits is a preselected number.

During the region growing process, an image data will be decomposed three data parts:

(1)corresponding error image data part

(2)corresponding discontinuity index image data part

(3)high-bits seed data part

When a new (neighbor) pixel $x_{j}$ is included in the region being grown, its difference $e_{j}$ with respect to its center pixel $x_{i}$ is stored as the pixel's "error" value using the Eq. (3).

$$
e_{j}=x_{j}-x_{i}+\text { error level }
$$

If only the first one of the two region growing criteria is satisfied, the discontinuity index of the pixel is incremented. The error value of the seed pixel of each region is defined as the value of its low (error bits) bits; the value of high ( $\mathrm{N}$-error bits) bits of the pixel is stored in a "high-bits seed data part," where $\mathrm{N}$ is the number of the $b /$ pixel in the original image data.

The above three data parts are used to fully recover the original image during the decoding process. The criteria of region growing during decoding are that 


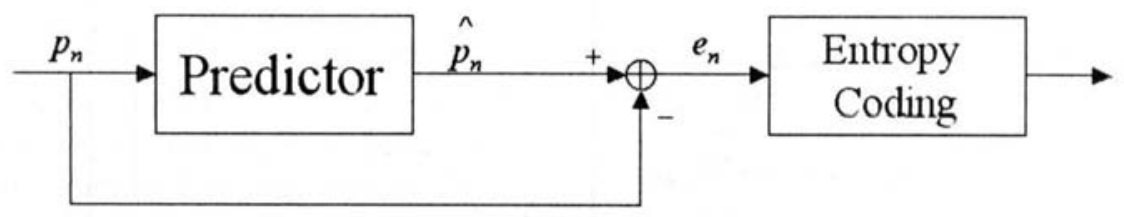

Fig. 1 Lossless Predictive Coding System.

a neighbor pixel under consideration to be included in a specific region must be not in any of the previously grown regions, and its discontinuity index equal to zero. When the criteria are satisfied for a pixel, its pixel value is restored as the sum of its error value $e_{j}$ and its center pixel value $x_{i}$ using Eq. (4):

$$
x_{j}=x_{1}+e_{j} \text {-error level }
$$

If only the first one of two criteria is satisfied, the discontinuity index of that pixel is decremented. And the "high-bits seed data part" is combined with the "error image data part" to recover the seed pixel value of each region.

\subsection{Segmentation Based Compression Model}

The segmentation technique - region growing is the first step for the proposed HCM method. Assume that there is a cluster of similar images $X=\left\{x_{1}, x_{2}, \ldots, x_{N}\right\}$ that we want to process. The corresponding median image $m$ is determined and treated as the input of region growing procedure. For a pixel $m_{i}$, its neighbor pixel is checked with the region growing conditions. The neighbor pixel $m_{i+1}$ is included in the region, if the conditions are satisfied. Furthermore, corresponding pixel is also included in the same region in each image that belong image cluster $X$. In other words, the pixel $x_{i, 1}$ and $x_{i+1,1}$ in the first image, $x_{i, 2}$ and $x_{i+1,2}$ in the second image, and $x_{i, N}$ and $x_{i+1, N}$ in the last image are processed at one time. $N$ clusters of corresponding error image data and highbits seed data are created when region growing procedure is completed, and only one cluster of discontinuity index is stored.

Exploit region growing technique to analyze the content of the median image which is determined by a cluster of similar images can group spatially connected pixels whose gray level are within a small dynamic range. The next stage is to use centroid method to predict the estimates of the corresponding error image data, and the difference between predict values and original data is then determined and stored. Eq. (5) is the modified centroid method formula.

$$
\begin{aligned}
& C_{e_{x_{i+1, k}}}=e_{m_{t+1}}+r_{e_{x_{i, k}}} \\
& r_{e_{x_{i, k}}}=e_{x_{i, k}}-e_{m_{i}}
\end{aligned}
$$

where $C_{e_{x_{i+1, k}}}$ is the estimate of the corresponding error image data at position $i+1$ in image $k, e_{m_{i+1}}$ is the corresponding error image data at position $i+1$ in the median image, and $e_{x_{i, k}}$ is the corresponding error image data at position $i+1$ in image $k$. The difference value at position $i+1$ in image $k$ is shown as following.

$$
D_{x_{i+1, k}}=e_{x_{i+1, k}}-C_{e_{x_{i+1 k}}}
$$

\section{EXPERIMENTAL RESULTS}

The main purpose of the proposed scheme (HCM) is to efficiently compress a cluster of similar images such as medical images by reducing the cluster redundancy further. In the following, three compression procedures are developed to evaluate the performance of our system. These three procedures include hybrid lossless compression process, hybrid lossy compression process, and progressive transmission process.

As described above, data compression technique is divided two parts: lossless compression technique and lossy compression technique. The lossless compression procedure mainly includes the stage of hybrid

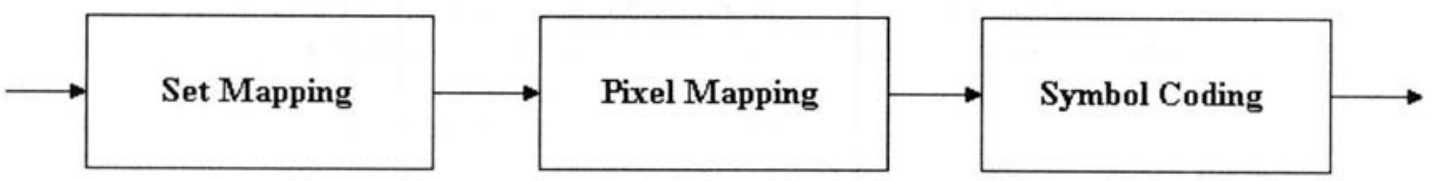

Fig. 2 Enhanced Compression Model. 
compression model (i.e., segmentation based centroid method) and the stage of entropy coding (i.e., LZW coding [9-10]). In the lossy compression procedure, here, besides the segmentation based centroid method and LZW coding techniques are used the same as lossless compression procedure, the wavelet transform and quantization technique are utilized for data reduction further.

Recently, encoder based on discrete wavelet transform become more and more popular in image compression due to its flexibility to nonstationary signals, the fast implementation, and the effective coding technique for wavelet coefficients. The principle behind wavelet transform is to represent a signal as a superposition of wavelets, which are formed by dilation and translation of a mother wavelet $\Psi(t)$, i.e.,

$$
W T_{x}(a, \tau)=\frac{1}{\sqrt{a}} \int x(t) \Psi^{\bullet}\left(\frac{t-\tau}{a}\right) d t=\left\langle x(t), \Psi_{a \tau}(t)>\right.
$$

With good frequency selectivity at lower frequencies and good time selectivity at higher frequencies, wavelet transform gives a spatial and frequency representation of signal. According to the fast algorithm proposed by Mallat [11], discrete wavelet transform can be implemented by a filter bank as shown in Fig. 3 . $h_{0}(n)$ and $h_{1}(n)$ are low-pass and high-pass analysis filters, and $g_{0}(n)$ and $g_{1}(n)$ are low-pass and high-pass synthesis filters, respectively. In order to implement perfect reconstruction, the filter coefficients should satisfy the criteria defined as Eq. (8).

$$
\begin{aligned}
& g_{1}(n)=(-1)^{n} h_{0}(-n+1) \\
& g_{0}(n)=(-1)^{n} h_{1}(-n+1)
\end{aligned}
$$

There are various extensions of the onedimension (1-D) wavelet transform to higher dimensions. Fig. 4 presents one stage in a wavelet decomposition of an image.

In a conventional way of viewing an image, the content of the image cannot be revealed until the transmission is almost over. Using the progression transmission technique, however, the resolution of an image increases gradually according to the amount of data transmitted. That is, progressive transmission of an image permits the initial reconstruction of an approximation followed by a gradual improvement of quality in image reconstruction. When the speed of the transmission or the bandwidth of the communication channel is relatively high compared to the image data size, the conventional method of viewing the image is not a problem. However, when the amount of image data is very large, the progressive transmission plays an important role in viewing or browsing the image. Since the image can be viewed progressively, a high

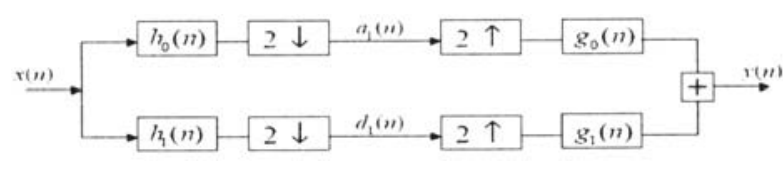

Fig. 3. Two-Channel filter banks for wavelet transform.

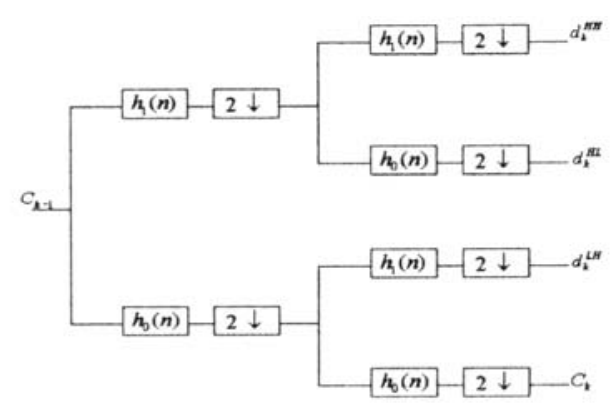

Fig. 4. Filter banks of 2-D DWT.

degree of effective compression ratio can be achieved by not transmitting the rest of the data when the image turns out to be of no interest.

A spatial-based approach for progressive transmission generates a cluster of image frames at different resolutions by means of subsampling or averaging. The top level of the pyramid initiates the transmission and it can be expended as the lower level of the structure is transmitted. Conventional pyramidal structure required one-third more space to build the hierarchy as illustrated in Fig. 5.

For example, HINT (Hierarchical Interpolation) is a variable resolution pyramid based on the subsampling [6]. In HINT, a subsampled version of the original image consisting of the pixels (symbol " 1 " shown in Fig. 6) is transmitted first. Next, the intermediate pixel (symbol " 2 " shown in Fig. 6) was estimated by linear interpolation of the four surrounding pixels (symbol "1" shown in Fig. 6). The estimation was rounded to the nearest integer to ensure the reversibility. Then, the difference between the estimated value and the actual pixel value was coded using variable length coding, like Huffman coding.

In HINT, only the interpolated pixels needed a residual in order to be reconstructed exactly, since the subsampled pixels are already exact. As a result, the total number of values that must be stored is equal to the number of original pixels. Fig. 7 shows that the LENA image through the HINT progressive reconstruction for five different resolutions.

In the experiments, four groups of medical images have been established for the purpose of evaluat- 


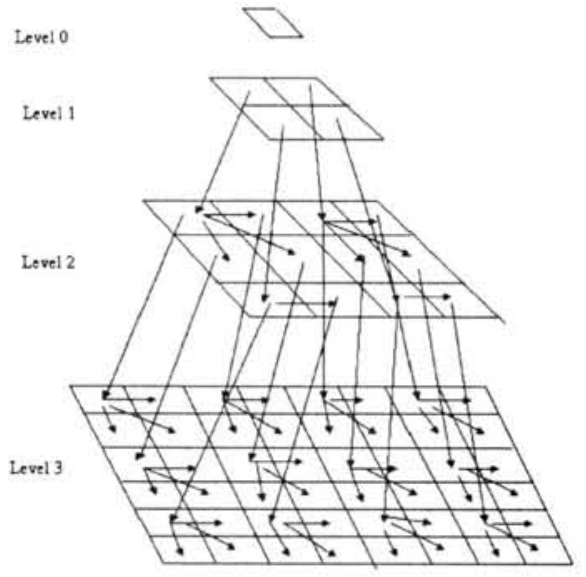

Fig. 5. Pyramidal structure.

\begin{tabular}{|l|l|l|l|l|l|l|l|l|}
\hline 1 & 5 & 3 & 5 & 1 & 5 & 3 & 5 & 1 \\
\hline 5 & 4 & 5 & 4 & 5 & 4 & 5 & 4 & 5 \\
\hline 3 & 5 & 2 & 5 & 5 & 5 & 2 & 5 & 3 \\
\hline 5 & 4 & 5 & 4 & 5 & 4 & 5 & 4 & 5 \\
\hline 1 & 5 & 3 & 5 & 1 & 5 & 3 & 5 & 1 \\
\hline 5 & 4 & 5 & 4 & 5 & 4 & 5 & 4 & 5 \\
\hline 3 & 5 & 2 & 5 & 3 & 5 & 2 & 5 & 3 \\
\hline 5 & 4 & 5 & 4 & 5 & 4 & 5 & 4 & 5 \\
\hline 1 & 5 & 3 & 5 & 1 & 5 & 3 & 5 & 1 \\
\hline
\end{tabular}

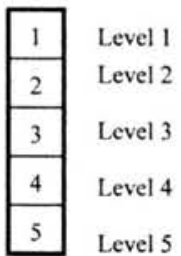

Fig. 6. HINT pyramid.

ing the system performance. Some of these images are shown in Fig. 8. Their content is summarized as below.

1. Brain CT images $\times 11,512 \times 512$ pixels, 8 bits/pixel gray scale

2. Chest CT images $\times 15,256 \times 256$ pixels, 8 bits/pixel gray scale

3. Ultrasound images $\times 15,256 \times 256$ pixels, 8 bits/pixel gray scale

4. Brain MRI T1 images $\times 15,256 \times 256$ pixels, 8 bits/pixel gray scale

Various tests are conducted to compare the efficiency of the proposed HCM model and traditional centroid method in compression ratio and peak-signalnoise-ratio (PSNR). Details of the comparison are described as below.

\subsection{Performance Comparison of the HCM and Centroid Method}

Clusters of similar medical image compressed by various error bit are tabulated in Table 1. According to different organization, texture etc., region growing technique analyze the content of image data and clas-

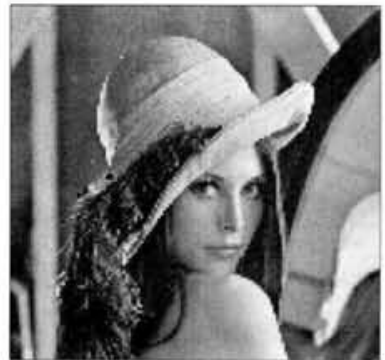

(a)

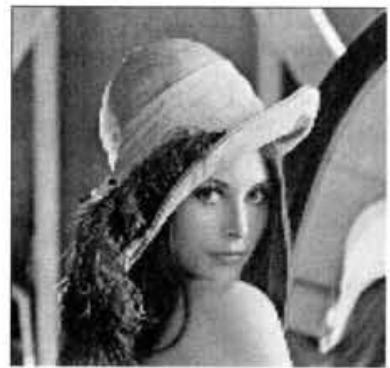

(c)

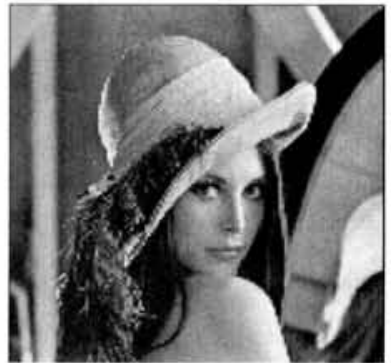

(b)

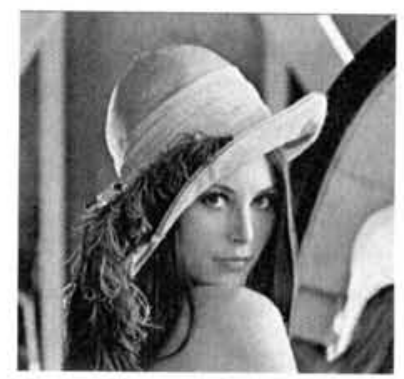

(e)

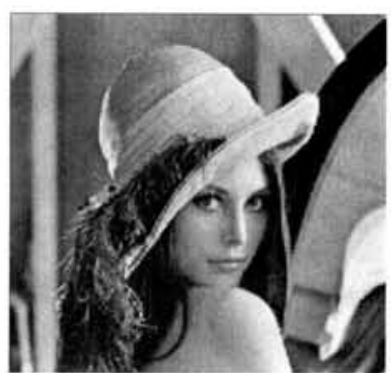

(d)

Fig. 7 HINT reconstructed image (a) level 1 (b) level 2 (c) level 3 (d) level 4 (e) level 5.

sify it become various region we expect. So there are different optimal values for different error bits that are the variables in region growing process. The following experiment will use the value 5 be the error bit.

Performance comparison results between HCM and centroid method are tabulated in Table 2. It shows that the performances using HCM are all better than centroid method. The improvement between traditional centroid method and HCM is $5.6 \%$ to $134.9 \%$.

\subsection{Performance Comparison for Compression Ratio and PSNR Using Wavelet Transform}

Five different wavelet mother functions [12] are used in the experiments. To compare with different qualities of images, quantization threshold value $T$ is set in the range of 5 50. Table 3 is the results for brain $\mathrm{CT}$ images. It shows that the performances are the best using wavelet mother function HAAR for brain CT image. However, in other experiments [13], using HAAR function are the worst results relatively for 


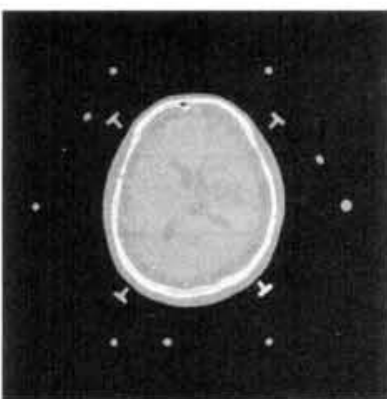

(a)

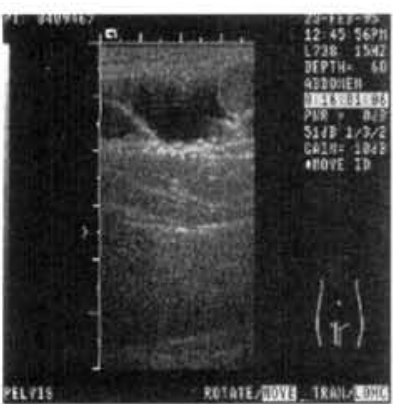

(c)

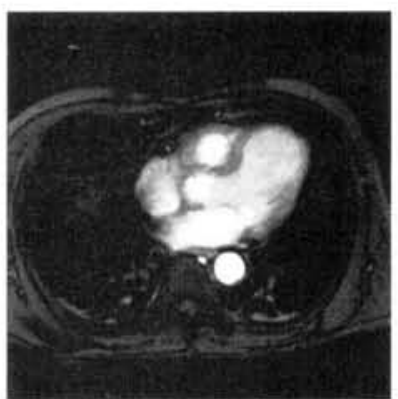

(b)

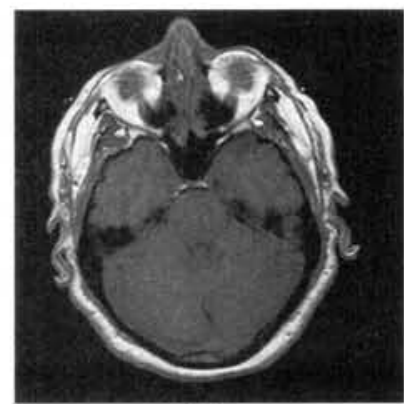

(d)
Fig. 8. Experimental image data set (a) One of brain CT images $\times 11,512 \times 512$ pixels, 8 bits/pixel gray scale (b) One of chest CT images $\times 15,256 \times 256$ pixels, 8 bits/pixel gray scale (c) One of ultrasound images $\times 15,256 \times 256$ pixels, 8 bits/pixel gray scale (d) One of brain MRI T1 images $\times 15,256 \times 256$ pixels, 8 bits/pixel gray scale.

chest CT images. In other words, the key point to obtain the best compression ratio is not the kind of the wavelet mother function. The critical point should be the image features such as contents, organization and texture etc. Reconstructed brain CT images using various wavelet mother functions is illustrated in Fig. 9. The result shows that the image quality is very close original ones

\subsection{Performance Comparison between Bit Rate and PSNR Using HINT}

HINT is a progressive transmission technique in spatial domain, and a set of brain CT images (i.e., 11 slices) is used as an example to illustrate the system performance. These images are decomposed and reconstructed with five different resolution levels. From the experimental results, we observe that the content of reconstructed image can be distinguished clearly with the progressive data part of level 1. Table 4 shows the comparison between bit rate and PSNR under different levels. The result shows that the progressive transmission using hybrid compression model through HINT can get good performance in low bit rate.

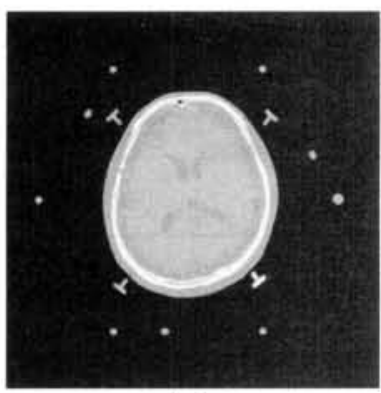

(a)

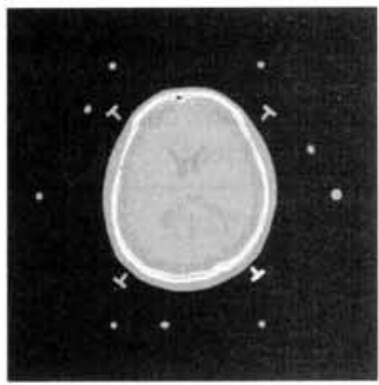

(c)

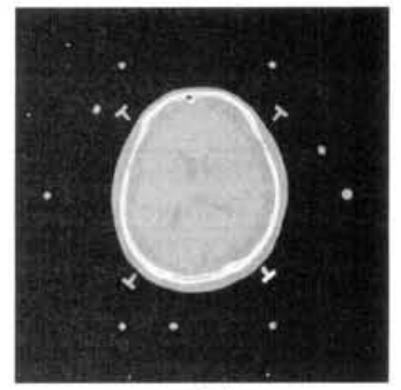

(b)

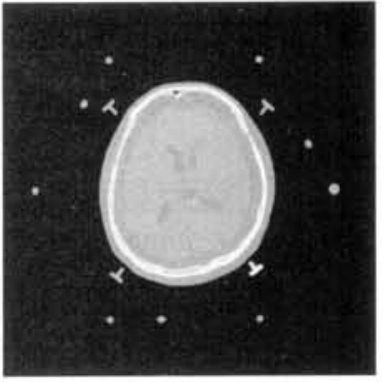

(d)

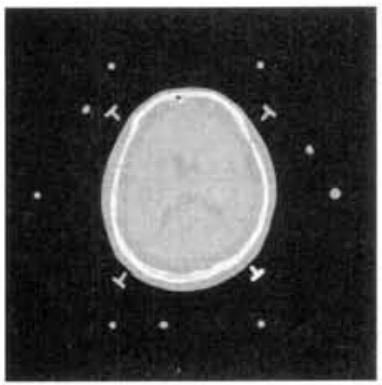

(e)

Fig. 9. Reconstructed brain CT image data using various wavelet mother function, $T=5$ (a) BIOR3.7 (b) COIF (c) DB4 (d) HAAR (e) SYM4.

\section{CONCLUSION}

In this paper, a new compression scheme (HCM) is proposed for compressing clusters of similar images. Compared with traditional still image compression technique whose goal is to reduce the three redundancies, similar images compression can decrease cluster redundancy further and thus improve the overall performance of the system. That is, the cooperation between $\mathrm{HCM}$ and progressive transmission technique can let similar images, especially medical images that have large amount data, transmission efficiently over network and achieve low bit rate. 
Table 1. Performance comparison between error bit and compression ratio.

\begin{tabular}{|c|c|c|c|c|c|}
\hline & \multicolumn{5}{|c|}{ Compression Ratio } \\
\cline { 2 - 6 } & error bit =6 & error bit = 5 & error bit =4 & error bit = 3 & error bit = 2 \\
\hline $\begin{array}{c}256 \text { 256Chest } \\
\text { CT image } \times 15\end{array}$ & 3.262 & 3.341 & 3.340 & 3.214 & 3.199 \\
\hline $\begin{array}{c}\text { 256X256Brain } \\
\text { MR T1 image } \times \\
15\end{array}$ & 1.646 & 1.677 & 1.679 & 1.675 & 1.637 \\
\hline $\begin{array}{c}512 X 512 B r a i n \\
\text { CT image } \times 11\end{array}$ & 17.078 & 17.213 & 18.346 & 17.165 & 17.155 \\
\hline
\end{tabular}

Table 2. Performance comparison between HCM and Centroid method.

\begin{tabular}{|c|c|c|c|}
\hline Image data set & $\begin{array}{c}\text { Correiation coeffi- } \\
\text { cient }\end{array}$ & Centroid Method & HCM \\
\cline { 3 - 4 } & 0.9411 & Compression Ratio & Compression Ratio \\
\hline $\begin{array}{c}256 \times 256 \text { Chest CT } \\
\text { image } \times 15\end{array}$ & 2.321 & 3.341 \\
\hline $\begin{array}{c}256 \times 256 \text { Brain MR } \\
\text { T1 image } \times 15\end{array}$ & 0.4770 & 1.588 & 1.677 \\
\hline $\begin{array}{c}512 \times 512 \text { Brain CT } \\
\text { image } \times 11\end{array}$ & 0.999 & 7.350 & 17.213 \\
\hline
\end{tabular}

Table 3. Performance comparison between compression ratio (CR) and PSNR using various wavelet functions for brain CT images.

\begin{tabular}{|c|c|c|c|c|c|c|c|c|c|c|c|}
\hline & $T$ & 05 & 10 & 15 & 20 & 25 & 30 & 35 & 40 & 45 & 50 \\
\hline \multirow{2}{*}{ BIOR } & CR & $\mathbf{1 7 . 3 4 9}$ & $\mathbf{2 1 . 7 6}$ & $\mathbf{2 8 . 0 3 3}$ & $\mathbf{3 3 . 1 2 4}$ & $\mathbf{3 6 . 2 6 5}$ & $\mathbf{3 9 . 3 3 0}$ & $\mathbf{4 2 . 4 4 9}$ & $\mathbf{4 4 . 3 2 6}$ & $\mathbf{4 5 . 7 8 3}$ & $\mathbf{4 7 . 6 6 9}$ \\
\cline { 2 - 10 } 3.7 & PSNR & $\mathbf{8 3 . 6 3 9}$ & $\mathbf{7 9 . 0 1 0}$ & $\mathbf{7 6 . 0 3 1}$ & $\mathbf{7 4 . 0 9 8}$ & $\mathbf{7 4 . 0 5 6}$ & $\mathbf{7 4 . 0 2 9}$ & $\mathbf{7 3 . 2 8 0}$ & $\mathbf{7 3 . 4 6 9}$ & $\mathbf{7 3 . 0 9 1}$ & $\mathbf{7 2 . 6 5 0}$ \\
\hline \multirow{2}{*}{ COIF 3 3 } & CR & $\mathbf{1 7 . 1 9 4}$ & $\mathbf{2 4 . 2 2 2}$ & $\mathbf{3 3 . 3 5 5}$ & $\mathbf{4 0 . 1 9 7}$ & $\mathbf{4 4 . 2 4 3}$ & $\mathbf{4 6 . 9 7 1}$ & $\mathbf{4 9 . 5 7 5}$ & $\mathbf{5 1 . 2 4 8}$ & $\mathbf{5 3 . 1 6 9}$ & $\mathbf{5 6 . 7 9 7}$ \\
\cline { 2 - 10 } & PSNR & $\mathbf{8 6 . 5 9 9}$ & $\mathbf{8 2 . 7 9 5}$ & $\mathbf{7 9 . 8 5 9}$ & $\mathbf{7 8 . 1 6 4}$ & $\mathbf{7 5 . 3 6 7}$ & $\mathbf{7 4 . 0 6 7}$ & $\mathbf{7 2 . 5 7 8}$ & $\mathbf{7 2 . 4 9 5}$ & $\mathbf{7 1 . 5 8 8}$ & $\mathbf{7 1 . 1 1 8}$ \\
\hline \multirow{2}{*}{ DB 4 4} & CR & $\mathbf{1 7 . 5 4 2}$ & $\mathbf{2 5 . 6 1 3}$ & $\mathbf{3 5 . 7 6 2}$ & $\mathbf{4 2 . 7 3 1}$ & $\mathbf{4 7 . 1 7 9}$ & $\mathbf{5 1 . 4 3 7}$ & $\mathbf{5 5 . 1 1 7}$ & $\mathbf{5 7 . 8 8 5}$ & $\mathbf{6 0 . 4 4 7}$ & $\mathbf{6 2 . 9 9 9}$ \\
\cline { 2 - 10 } & PSNR & $\mathbf{8 5 . 1 9 0}$ & $\mathbf{8 0 . 6 2 3}$ & $\mathbf{7 8 . 0 2 8}$ & $\mathbf{7 6 . 1 3 8}$ & $\mathbf{7 5 . 2 9 0}$ & $\mathbf{7 3 . 8 0 9}$ & $\mathbf{7 3 . 5 8 7}$ & $\mathbf{7 3 . 5 0 7}$ & $\mathbf{7 3 . 3 6 1}$ & $\mathbf{7 2 . 9 7 2}$ \\
\hline \multirow{2}{*}{ HAAR } & CR & $\mathbf{2 0 . 4 2 4}$ & $\mathbf{3 0 . 3 0 2}$ & $\mathbf{3 4 . 7 6 6}$ & $\mathbf{4 8 . 9 6 2}$ & $\mathbf{5 2 . 7 7 8}$ & $\mathbf{5 5 . 8 8 0}$ & $\mathbf{5 8 . 8 3 1}$ & $\mathbf{6 0 . 9 5 2}$ & $\mathbf{6 3 . 4 0 4}$ & $\mathbf{6 8 . 2 0 7}$ \\
\cline { 2 - 9 } & PSNR & $\mathbf{8 7 . 5 2 5}$ & $\mathbf{8 4 . 8 5 3}$ & $\mathbf{8 3 . 0 9 5}$ & $\mathbf{8 0 . 3 8 7}$ & $\mathbf{7 8 . 2 1 8}$ & $\mathbf{7 4 . 9 6 9}$ & $\mathbf{7 4 . 6 1 5}$ & $\mathbf{7 3 . 4 3 3}$ & $\mathbf{7 2 . 1 2 4}$ & $\mathbf{7 1 . 5 4 4}$ \\
\hline \multirow{2}{*}{ SYM 4 4 } & CR & $\mathbf{1 7 . 3 9 0}$ & $\mathbf{2 4 . 7 2 1}$ & $\mathbf{3 5 . 0 1 2}$ & $\mathbf{4 2 . 9 6 9}$ & $\mathbf{4 8 . 0 4 3}$ & $\mathbf{5 1 . 9 8 9}$ & $\mathbf{5 4 . 5 7 1}$ & $\mathbf{5 7 . 3 4 6}$ & $\mathbf{5 7 . 8 3 4}$ & $\mathbf{5 9 . 4 6 0}$ \\
\cline { 2 - 9 } & PSNR & $\mathbf{8 4 . 8 3 5}$ & $\mathbf{7 9 . 7 5 8}$ & $\mathbf{7 8 . 6 8 9}$ & $\mathbf{7 7 . 9 5 3}$ & $\mathbf{7 5 . 1 9 0}$ & $\mathbf{7 4 . 9 4 7}$ & $\mathbf{7 4 . 6 5 1}$ & $\mathbf{7 3 . 8 1 1}$ & $\mathbf{7 3 . 6 7 6}$ & $\mathbf{7 2 . 6 3 2}$ \\
\hline
\end{tabular}

Table 4. Performance comparison between PSNR and bit rate using HINT progressive transmission technique and HCM.

\begin{tabular}{|c|c|c|c|c|c|}
\hline & Level 1 & Level 2 & Level 3 & Level 4 & Level 5 \\
\hline PSNR & 79.4705 & 80.2836 & 81.2625 & 85.520 & $\cdots$ \\
\hline Bit rate & 0.0414 & 0.1332 & 0.2951 & 0.5499 & 1.0229 \\
\hline
\end{tabular}

\section{ACKNOWLEDGEMENT}

This work was supported by Chang Gung Memorial Hospital, under Grant CMRP1243.

\section{REFERENCE}

1. Y. Li, J. Kim and N. Al-Shamakhi, "Image compression using transformed vector quantization". Image and Vision Computing 2002; 20; 37-45.

2. M. Miatvik and I. Hovig, "Reversible Compression of MR Images." IEEE Trans. Medical Imaging $1999 ; 18 ; 9 ; 795-800$.

3. H. Jian-Hong et al., "Multispectral Code Excited Linear Prediction Coding and Its Application in Magnetic Resonance Images". IEEE Trans. Image Processing vol6, noll, pp 1555-1566, November 
1997.

4. K. Karadimitriou et al., "Centroid method for compressing sets of similar images". Pattern Recognition Letters vol.9, no.7, pp. 585-593, 1998.

5. K. Tzon Progressive image transmission: A review and comparison of techniques." Opt Eng., Vol.26, pp. $1987 ; 26 ; 581-589$, July 1987.

6. Y.S. Kim and W.Y. Kim, "ReversibleDecorrelation Method for Progressive Transmission of 3-D Medical Image. vol.17, no3, June 1998.

7. P. Roos et al., "Reversible intraframe compression of medical images." IEEE Trans. Medical Imaging vol.7, pp.328-336, Dec. 1998.

8. S. Liang and R.M. Rangayyan "A Segmentation Based Lossless Image Coding Method for HighResolution Medical Image Compression." IEEE Trans. Medical Imaging, vol.16, no.3, pp301-307, June 1997.

9. J. Ziv and A. Lempel "A universal algorithm for sequential data compression. IEEE Trans. information theory" vol. IT-23, no.3, pp.337-343. May 1977.

10.J. Ziv and A. Lempel., "Compression of individual sequences via variable-rate coding". IEEE Trans. information theory, vol.24, no 5, pp. 530-536, Sep. 1978.

11.S. Mallat., "A theory for multiresolution signal decomposition - The wavelet representation." IEEE Trans. Pattern Anal. Mach. Intel., vol.11, pp.674693, July 1989.

12.I. Daubechies "Orthogonal bases of compactly supported wavelets." Commun. Pure Appl. Math., vol. XLI, pp. 909-996, 1988.

13.R.F. Wu, "Hybrid compression model for sets of similar images and its application to progressive transmission, Master Thesis, Chang Gung University, 2000. 